\title{
Lang wirksames Anticholinergikum hilft Patienten aus dem Morgen-Tief
}

- Seit Anfang Oktober bietet BerlinChemie mit Bretaris ${ }^{\circledR}$ Genuair $^{\circledR}$ (Wirkstoff: Aclidinium) ein neues lang wirksames inhalatives Anticholinergikum zur COPDTherapie an. Bei zweimal täglicher Anwendung wirkt es zuverlässig über 24 Stunden und lindert vor allem nächtliche und morgendliche Symptome, unter denen COPDPatienten besonders leiden.

COPD-Beschwerden sind ungleich über den Tag verteilt: Gerade Patienten mit schwerer Erkrankung der Stadien III und IV leiden vor allem nachts und in den frühen Morgenstunden, berichtete Prof. Heinrich Worth, Fürth. Der Tag dieser Patienten beginnt mit Husten, Auswurf und Atemnot Aufstehen, Waschen und Anziehen werden zur Qual. Die Lebensqualität ist massiv be- einträchtigt. „Wir müssen diesen Patienten helfen, besser in den Tag zu kommen", sagte Worth.

\section{Schnelle systemische Elimination}

Anticholinergika sind laut Worth bei COPD Basismedikation in den meisten Krankheitsstadien. Aclidinium hat eine hohe Affinität zum relevanten Muskarin3-Rezeptor, wird aber systemisch schnell eliminiert. In Studien zeigte es gegenüber Placebo eine klinisch relevante Verbesserung der Lungenfunktion sowie eine Reduktion der prognostisch bedeutsamen Exazerbationen um $33 \%$, so Worth. Damit einher ging eine Linderung der Symptomatik, insbesondere der Atemnot, eine Verbesserung der Le- bensqualität sowie Reduktion der Notfallmedikation. Gerade nachts und in den Morgenstunden war die Wirkung auf die Symptomatik ausgeprägt.

Bretaris $^{\circledR}$ wird zweimal täglich über den Genuair ${ }^{\circledast}$ Pulverinhalator atemzuggesteuert inhaliert. Bei dem Multi-Dosis-Inhalator handelt es sich um eine Weiterentwicklung des Novolizers, der intuitiv, einfach und sicher zu bedienen ist und Anwendungsfehler minimiert.

\footnotetext{
- Dr. med. Dirk Einecke

Quelle: Pressekonferenz ${ }_{\text {"Bretaris }}{ }^{\oplus}$ Genuair $^{\oplus}$

- der neue langwirksame Antagonist muskarinischer Acetylcholinrezeptoren (LAMA) in der COPD-Therapie“, Berlin, Oktober 2012 (Veranstalter: Berlin-Chemie)
}

\section{Neuer Meningokokken-Konjugatimpfstoff}

\section{Hohe Antikörper-Persistenz schon bei Kindern ab einem Jahr}

- Im April 2012 wurde ein tetravalenter Meningokokken-Konjugatimpfstoff (Nimenrix ${ }^{\circledast}$ ) zugelassen. Er ist indiziert für „die aktive Immunisierung von Personen ab dem vollendeten zwölften Lebensmonat gegen invasive Meningokokken-Erkrankungen, verursacht durch Neisseria meningitidis der Serogruppen A, C, W-135 und Y." Er enthält jeweils $5 \mu \mathrm{g}$ der vier Polysaccharid-Antigene, gekoppelt an $44 \mu \mathrm{g}$ TetanusToxoid. Damit steht europaweit erstmals ein konjugierter Impfstoff gegen vier der sechs häufigsten Meningokokken-Serogruppen schon für Kinder im zweiten Lebensjahr zur Verfügung. Auch für ältere Kinder, Jugendliche und Erwachsene ist der Impfstoff geeignet.

"Studien haben für Nimenrix schon bei Impflingen ab einem Jahr Immunogenität und eine hohe Antikörper-Persistenz gezeigt", sagte Prof. Markus Knuf, Wiesbaden. So wurden die Antikörpertiter gegen Meningokokken der Serogruppen A, C, W-135 und $Y$ bei Kindern im Alter von zwölf bis 23

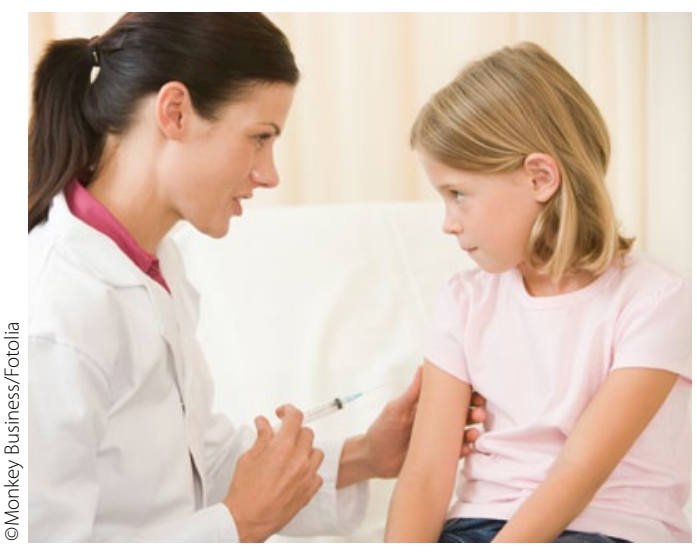

Der neue Impfstoff ist auch für Kinder, Jugendliche und Erwachsene geeignet.

Monaten sechs bis acht Wochen nach der Impfung gemessen. Von den mit dem neuen Konjugatimpfstoff geimpften Kindern erreichten 99,7\% (A, C) bzw. 100\% (W-135, $Y)$ einen bakteriziden Antikörper-Titer von mindestens 1:8. Mit einem konjugierten MenC-Vergleichsimpfstoff erzielten 97,5\% der Kinder dieser Altersgruppe diesen Titer.
Zwei Jahre nach Immunisierung mit dem neuen Konjugatimpfstoff fanden sich bakterizide Antikörpertiter noch bei $97,8 \%$, 88,2\%, 98,9\% bzw. 97,9\% der Impflinge (A, C, W-135, Y). Mit dem Vergleichsimpfstoff gegen MenC waren es $69 \%$.

Auch hinsichtlich der Serokonversionsrate bei Kindern ab zwei Jahren war der neue Impfstoff den Vergleichsimpfstoffen nicht unterlegen: Die Rate lag einen Monat nach Impfung mit dem Konjugatimpfstoff vs. ACWY-Polysaccharidimpfstoff bei 88,6 vs. $65,5 \%, 95,5$ vs. $89,6 \%, 97,4$ vs. $82,5 \%$ bzw. 92,5 vs. $68,6 \%$

\footnotetext{
- Simone Reisdorf Quelle: Launch-Pressekonferenz „Neue 4-valenter Meningokokken-Konjugatimpfstoff ( $A$, C, W-135, Y) für die ganze Familie: Impfung für Kleinkinder und Schutz auf Reisen", München, Juni 2012 (Veranstalter: GlaxoSmithKline)
} 